\title{
The Solution of Nonlinear Fourth-Order Differential Equation with Integral Boundary Conditions
}

\author{
Yanli Fu and Huanmin Yao \\ School of Mathematics and Sciences, Harbin Normal University, Harbin, \\ Heilongjiang 150025, China \\ Correspondence should be addressed to Huanmin Yao; hsdhmyao823@126.com
}

Received 20 January 2014; Accepted 22 March 2014; Published 20 May 2014

Academic Editor: Donghai Ji

Copyright ( $\odot 2014$ Y. Fu and H. Yao. This is an open access article distributed under the Creative Commons Attribution License, which permits unrestricted use, distribution, and reproduction in any medium, provided the original work is properly cited.

An iterative algorithm is proposed for solving the solution of a nonlinear fourth-order differential equation with integral boundary conditions. Its approximate solution $u_{n}(x)$ is represented in the reproducing kernel space. It is proved that $u_{n}(x)$ converges uniformly to the exact solution $u(x)$. Moreover, the derivatives of $u_{n}(x)$ are also convergent to the derivatives of $u(x)$. Numerical results show that the method employed in the paper is valid.

\section{Introduction}

Integral boundary conditions for evolution problems have various applications in chemical engineering, thermoelasticity, underground water flow, and population dynamics. In fact, boundary value problems (BVPs) involving integral boundary conditions have received considerable attention. For BVPs with integral boundary conditions and comments on their importance, we refer the reader to the papers by Gallardo [1], Karakostas and Tsamatos [2], Lomtatidze and Malaguti [3], and the references therein.

In this paper, we study the nonlinear fourth-order differential equation with integral boundary conditions in the reproducing kernel space:

$$
\begin{gathered}
u^{(4)}(x)-\lambda f(x, u(x))=0, \quad 0<x<1, \\
u(0)=u(1)=\int_{0}^{1} h_{1}(s) u(s) d s, \\
u^{\prime \prime}(0)=u^{\prime \prime}(1)=\int_{0}^{1} h_{2}(s) u^{\prime \prime}(s) d s,
\end{gathered}
$$

where $\lambda$ is a nonnegative constant. The existence of (1) was obtained in $[4,5]$. However, the literature of numerical analysis contains little on the solution of (1).
In the reproducing kernel spaces, many classical problems such as population models and complex dynamics have been solved $[6,7]$. For more details of the reproducing kernel spaces, we refer the readers to [8-11]. In [8-11], twopoint BVPs were solved in the reproducing kernel space which satisfied two-point boundary conditions. In this paper, however, we can solve integral boundary problems in the reproducing kernel space which satisfies integral boundary conditions. Reference [12] investigated the existence and multiplicity of symmetric positive solutions for a class of Laplacian fourth-order differential equations with integral boundary conditions. The arguments were based upon a specially constructed cone and the fixed point theory for cones. In [13], the authors are concerned with a new algorithm for giving the analytical and approximate solutions of a class of fourth-order in the new reproducing kernel space. Theorem on the completeness of the system of eigenvector and associated vectors of the operator were proved. Based upon a specially constructed cone and the fixed point theory in a cone, [14] established various results on the existence and nonexistence of symmetric positive solutions to fourth-order boundary value problems with integral boundary conditions.

In the paper, the representation of the exact and approximate solutions of (1) in the reproducing kernel space is 
given. The advantages of this method are as follows. First, the conditions for determining solution in (1) can be imposed on the reproducing kernel space and therefore the reproducing kernel satisfying the conditions for determining solution can be calculated. We will use the kernel to solve problems. Second, the iterative sequence $u_{n}(x)$ of approximate solutions converges in $C^{4}$ to the solution $u(x)$.

This paper is organized as follows. Several reproducing spaces and a linear operator are introduced in Section 2. Section 3 provides the main results; the exact and approximate solutions of (1) and an iterative method are developed for the kind of problems in the reproducing kernel space. We verify that the approximate solution converges to the exact solution uniformly. Some numerical experiments are illustrated in Section 4. Finally, Section 5 is the conclusions.

\section{Preliminaries}

2.1. The Reproducing Kernel Space $W_{5}[0,1]$. The inner product space $W_{5}[0,1]$ is defined as follows: $W_{5}[0,1]=\{u(x) \mid$ $u^{(4)}(x)$ is a absolutely continuous real-valued function, $u^{(5)}(x) \in L^{2}[0,1], u(0)=u(1)=\int_{0}^{1} h_{1}(x) u(x) d x, u^{\prime \prime}(0)=$ $\left.u^{\prime \prime}(1)=\int_{0}^{1} h_{2}(x) u^{\prime \prime}(x) d x\right\}$.
The inner product and norm in $W_{5}[0,1]$ are defined, respectively, by

$$
\begin{gathered}
\langle u(x), v(x)\rangle_{W_{5}}=\sum_{i=0}^{4} u^{(i)}(0) v^{(i)}(0)+\int_{0}^{1} u^{(5)}(x) v^{(5)}(x) d x \\
\|u\|_{W_{5}}=\sqrt{\langle u, u\rangle_{W_{5}}},
\end{gathered}
$$

where $u, v \in W_{5}[0,1]$.

Theorem 1. The space $W_{5}[0,1]$ is a complete reproducing kernel space. That is, for each fixed $x \in[0,1]$, there exists $K_{5}(y, x) \in W_{5}[0,1]$, such that $\left\langle u(y), K_{5}(y, x)\right\rangle_{W_{5}}=u(x)$ for any $u(y) \in W_{5}[0,1]$ and $y \in[0,1]$. The reproducing kernel $K_{5}(y, x)$ can be written as

$$
K_{5}(y, x)= \begin{cases}\sum_{i=1}^{10} a_{i}(x) y^{i-1}-\sum_{i=1}^{2} c_{i}(x) H_{i}(y), & y \leq x \\ \sum_{i=1}^{10} b_{i}(x) y^{i-1}-\sum_{i=1}^{2} c_{i}(x) H_{i}(y), & y>x\end{cases}
$$

where

$$
\begin{aligned}
& H_{1}(y)=\int_{0}^{y} \int_{0}^{y} \int_{0}^{y} \int_{0}^{y} \int_{0}^{y} \int_{0}^{y} \int_{0}^{y} \int_{0}^{y} \int_{0}^{y} \int_{0}^{y} h_{1}(y) d y d y d y d y d y d y d y d y d y d y \\
& H_{2}(y)=\int_{0}^{y} \int_{0}^{y} \int_{0}^{y} \int_{0}^{y} \int_{0}^{y} \int_{0}^{y} \int_{0}^{y} \int_{0}^{y} \int_{0}^{y} \int_{0}^{y} h_{2}^{\prime \prime}(y) d y d y d y d y d y d y d y d y d y d y .
\end{aligned}
$$

$a_{i}(x), b_{i}(x)(i=1,2, \ldots 10)$ and $c_{1}(x), c_{2}(x)$, which are related to $h_{1}(x)$ and $h_{2}(x)$, will be given in the following proof.

Proof. (i) The proof of the completeness and reproducing property of $W_{5}[0,1]$ is similar to the proof of Theorem 1.3.1 in [15].

(ii) Now, let us find out the expression form of the reproducing kernel function $K_{5}(y, x)$ in $W_{5}[0,1]$.

Through several integration by parts for (2), we have

$$
\begin{aligned}
\langle u(y), & \left.K_{5}(y, x)\right\rangle_{W_{5}} \\
= & \sum_{i=0}^{4} u^{(i)}(0)\left[\partial_{y}^{i} K_{5}(0, x)-(-1)^{4-i} \partial_{y}^{9-i} K_{5}(0, x)\right] \\
& +\sum_{i=0}^{4}(-1)^{4-i} u^{(i)}(1) \partial_{y}^{9-i} K_{5}(1, x) \\
& -\int_{0}^{1} u(y) \partial_{y}^{10} K_{5}(y, x) d y,
\end{aligned}
$$

and since $u(x) \in W_{5}[0,1]$, it follows that

$$
\begin{gathered}
u(0)=u(1)=\int_{0}^{1} h_{1}(x) u(x) d x, \\
u^{\prime \prime}(0)=u^{\prime \prime}(1)=\int_{0}^{1} h_{2}(x) u^{\prime \prime}(x) d x .
\end{gathered}
$$

Then, we have

$$
\begin{aligned}
\langle u(y), & \left.K_{5}(y, x)\right\rangle_{W_{5}} \\
= & \sum_{i=0}^{4} u^{(i)}(0)\left[\partial_{y}^{i} K_{5}(0, x)-(-1)^{4-i} \partial_{y}^{9-i} K_{5}(0, x)\right] \\
& +\sum_{i=0}^{4}(-1)^{4-i} u^{(i)}(1) \partial_{y}^{9-i} K_{5}(1, x) \\
& -\int_{0}^{1} u(y) \partial_{y}^{10} K_{5}(y, x) d y \\
& +c_{1}\left[u(0)-\int_{0}^{1} h_{1}(y) u(y) d y\right] \\
& +c_{2}\left[u^{\prime \prime}(0)-\int_{0}^{1} h_{2}(y) u^{\prime \prime}(y) d y\right] \\
= & \sum_{i=0}^{4} u^{(i)}(0)\left[\partial_{y}^{i} K_{5}(0, x)-(-1)^{4-i} \partial_{y}^{9-i} K_{5}(0, x)\right] \\
& +\sum_{i=0}^{4}(-1)^{4-i} u^{(i)}(1) \partial_{y}^{9-i} K_{5}(1, x)
\end{aligned}
$$




$$
\begin{gathered}
-\int_{0}^{1} u(y) \partial_{y}^{10} K_{5}(y, x) d y \\
+c_{1}\left[u(0)-\int_{0}^{1} h_{1}(y) u(y) d y\right] \\
+c_{2}\left[u^{\prime \prime}(0)-u^{\prime}(1) h_{2}(1)+u^{\prime}(0) h_{2}(0)\right. \\
+h_{2}^{\prime}(1) u(1)-h_{2}^{\prime}(0) u(0) \\
\left.-\int_{0}^{1} h_{2}^{\prime \prime}(y) u(y) d y\right] .
\end{gathered}
$$

Note that the property of the reproducing kernel $\left\langle u(y), K_{5}(y, x)\right\rangle_{W_{5}}=u(x)$ and $u(0)=u(1), u^{\prime \prime}(0)=u^{\prime \prime}(1)$, and $K_{5}(y, x)$ is the solution of the following generalized differential equation:

$$
\begin{gathered}
-\left(\partial_{y}^{10} K_{5}(y, x)+c_{1} h_{1}(y)+c_{2} h_{2}^{\prime \prime}(y)\right)=\delta(y-x), \\
K_{5}(0, x)-\partial_{y}^{9} K_{5}(0, x)+c_{1}+\partial_{y}^{9} K_{5}(1, x) \\
-c_{2} h_{2}^{\prime}(0)+c_{2} h_{2}^{\prime}(1)=0 \\
\partial_{y}^{1} K_{5}(0, x)+\partial_{y}^{8} K_{5}(0, x)+c_{2} h_{2}(0)=0 \\
\partial_{y}^{2} K_{5}(0, x)-\partial_{y}^{7} K_{5}(0, x)+c_{2}+\partial_{y}^{7} K_{5}(1, x)=0, \\
\partial_{y}^{3} K_{5}(0, x)+\partial_{y}^{6} K_{5}(0, x)=0 \\
\partial_{y}^{4} K_{5}(0, x)-\partial_{y}^{5} K_{5}(0, x)=0 \\
-\partial_{y}^{8} K_{5}(1, x)-c_{2} h_{2}(1)=0 \\
-\partial_{y}^{6} K_{5}(1, x)=0 \\
\partial_{y}^{5} K_{5}(1, x)=0
\end{gathered}
$$

$$
\begin{aligned}
& H_{1}(y)=\int_{0}^{y} \int_{0}^{y} \int_{0}^{y} \int_{0}^{y} \int_{0}^{y} \int_{0}^{y} \int_{0}^{y} \int_{0}^{y} \int_{0}^{y} \int_{0}^{y} h_{1}(y) d y d y d y d y d y d y d y d y d y d y, \\
& H_{2}(y)=\int_{0}^{y} \int_{0}^{y} \int_{0}^{y} \int_{0}^{y} \int_{0}^{y} \int_{0}^{y} \int_{0}^{y} \int_{0}^{y} \int_{0}^{y} \int_{0}^{y} h_{2}^{\prime \prime}(y) d y d y d y d y d y d y d y d y d y d y .
\end{aligned}
$$

Now, we are ready to calculate the coefficient $a_{i}(x), b_{i}(x)(i=$ $1,2, \ldots, 10)$ and $c_{1}(x), c_{2}(x)$.

Since

$$
-\left(\partial_{y}^{10} K_{5}(y, x)+c_{1} h_{1}(y)+c_{2} h_{2}^{\prime \prime}(y)\right)=\delta(y-x),
$$

we have

$$
\begin{gathered}
\partial_{y}^{k} K_{5}(x+0, x)=\partial_{y}^{k} K_{5}(x-0, x), \quad k=0,1,2, \ldots, 8, \\
\partial_{y}^{9} K_{5}(x-0, x)-\partial_{y}^{9} K_{5}(x+0, x)=1 .
\end{gathered}
$$

While $y \neq x$, it is easy to know that $K_{5}(y, x)$ is the solution of the following constant linear homogeneous differential equation with 10 orders; that is,

$$
-\left(\partial_{y}^{10} K_{5}(y, x)+c_{1} h_{1}(y)+c_{2} h_{2}^{\prime \prime}(y)\right)=0,
$$

with the following boundary conditions:

$$
\begin{gathered}
K_{5}(0, x)-\partial_{y}^{9} K_{5}(0, x)+c_{1}+\partial_{y}^{9} K_{5}(1, x) \\
-c_{2} h_{2}^{\prime}(0)+c_{2} h_{2}^{\prime}(1)=0 \\
\partial_{y}^{1} K_{5}(0, x)+\partial_{y}^{8} K_{5}(0, x)+c_{2} h_{2}(0)=0, \\
\partial_{y}^{2} K_{5}(0, x)-\partial_{y}^{7} K_{5}(0, x)+c_{2}+\partial_{y}^{7} K_{5}(1, x)=0, \\
\partial_{y}^{3} K_{5}(0, x)+\partial_{y}^{6} K_{5}(0, x)=0 \\
\partial_{y}^{4} K_{5}(0, x)-\partial_{y}^{5} K_{5}(0, x)=0 \\
-\partial_{y}^{8} K_{5}(1, x)-c_{2} h_{2}(1)=0 \\
-\partial_{y}^{6} K_{5}(1, x)=0 \\
\partial_{y}^{5} K_{5}(1, x)=0 .
\end{gathered}
$$

We know that (9) has the characteristic equation $\lambda^{10}=0$, and the eigenvalue $\lambda=0$ is a root whose multiplicity is 10 . Therefore, the general solution of (9) is

$$
K_{5}(y, x)= \begin{cases}\sum_{i=1}^{10} a_{i}(x) y^{i-1}-\sum_{i=1}^{2} c_{i}(x) H_{i}(y), & y \leq x, \\ \sum_{i=1}^{10} b_{i}(x) y^{i-1}-\sum_{i=1}^{2} c_{i}(x) H_{i}(y), & y>x,\end{cases}
$$

where 
The above 10 equations in (12) and (15) provided 10 conditions for solving the coefficients $a_{i}(x), b_{i}(x)(i=1,2, \ldots 10)$ in (11). Noting that (10) and (16) provided 12 boundary conditions, we have 22 equations, that is, (12), (15), (10), and (16). It is easy to know that these 22 equations are linear equations with the variables $a_{i}(x), b_{i}(x)(i=1,2, \ldots, 10)$ and $c_{1}(x), c_{2}(x)$. Therefore, $a_{i}(x), b_{i}(x)(i=1,2, \ldots, 10)$ and $c_{1}(x), c_{2}(x)$ could be calculated by many methods. As long as the coefficients $a_{i}(x), b_{i}(x), i=1,2, \ldots, 10$, and $c_{1}(x), c_{2}(x)$ are known, the exact expression of the reproducing kernel function $K_{5}(y, x)$ could be calculated from (11).

2.2. The Reproducing Kernel Space $W_{1}[0,1]$. The inner product space is defined by $W_{1}[0,1]=\{u(x) \mid u$ is a absolutely continuous real-value function, $\left.u^{\prime} \in L^{2}[0,1]\right\}$.

The inner product and norm in $W_{1}[0,1]$ are given, respectively, by

$$
\begin{gathered}
\langle u(x), v(x)\rangle_{W_{1}}=u(0) v(0)+\int_{0}^{1} u^{\prime}(x) v^{\prime}(x) d x, \\
\|u\|_{W_{1}}=\sqrt{\langle u, u\rangle_{W_{1}}},
\end{gathered}
$$

where $u(x), v(x) \in W_{1}[0,1]$. In [15], it has been proved that $W_{1}[0,1]$ is also a complete reproducing kernel space and its reproducing kernel is

$$
K_{1}(y, x)= \begin{cases}1+y, & y \leq x \\ 1+x, & y>x\end{cases}
$$

2.3. Introduction into a Linear Operator. Let $L u=u^{(4)}$ and $L: W_{5}[0,1] \rightarrow W_{1}[0,1]$; then (1) can be converted into the form as follows:

$$
L u=F(x, u(x)), \quad 0<x<1,
$$

where $u(x) \in W_{5}[0,1]$ and $F(x, u(x))=\lambda f(x, u(x)) \in$ $W_{1}[0,1]$ as $u(x) \in W_{1}[0,1]$. Therefore, we have

$$
\begin{gathered}
u(0)=u(1)=\int_{0}^{1} h_{1}(x) u(x) d x, \\
u^{\prime \prime}(0)=u^{\prime \prime}(1)=\int_{0}^{1} h_{2}(x) u^{\prime \prime}(x) d x .
\end{gathered}
$$

It is easy to prove that $L$ is a bounded linear operator.

Now, we construct an orthogonal function system. Let $\varphi_{i}(x)=K_{1}\left(x, x_{i}\right)$ and $\psi_{i}(x)=L^{*} \varphi_{i}(x)$, where $L^{*}$ is the conjugate operator of $L$. In terms of the properties of reproducing kernel $K_{1}(y, x)$, one obtains

$$
\begin{aligned}
\left\langle u(x), \psi_{i}(x)\right\rangle_{W_{5}}=\left\langle L u(x), \varphi_{i}(x)\right\rangle_{W_{1}} & =L u\left(x_{i}\right), \\
i & =1,2, \ldots
\end{aligned}
$$

The normal orthogonal system of functions $\left\{\bar{\psi}_{i}(x)\right\}_{i=1}^{\infty}$ in $W_{5}[0,1]$ can be derived from Gram-Schmidt orthogonalization process of $\left\{\psi_{i}(x)\right\}_{i=1}^{\infty}$ :

$$
\bar{\psi}_{i}(x)=\sum_{k=1}^{i} \beta_{i k} \psi_{k}(x),
$$

where $\beta_{i k}$ are orthogonalization coefficients, $\beta_{i i}>0, i=$ $1,2, \ldots$.

We collect two lemmas in [9] for future use.

Lemma 2. If $\left\{x_{i}\right\}_{i=1}^{\infty}$ is dense on $[0,1]$, then $\left\{\psi_{i}(x)\right\}_{i=1}^{\infty}$ is a complete system of $W_{5}[0,1]$ and $\psi_{i}(x)=\left.L_{y} K_{5}(y, x)\right|_{y=x_{i}}$. The subscript $y$ by the operator $L$ indicates that the operator $L$ applies to the function of $y$.

Lemma 3. If $u(x) \in W_{5}[0,1]$, then there exists $M_{1}>0$, such that

$$
\|u\|_{C^{4}[0,1]} \leq M_{1}\|u\|_{W_{5}}
$$

where $\|u\|_{C^{4}[0,1]}=\max _{x \in[0,1]}\left(|u(x)|+\left|u^{\prime}(x)\right|+\left|u^{\prime \prime}(x)\right|+\right.$ $\left.\left|u^{\prime \prime \prime}(x)\right|+\left|u^{(4)}(x)\right|\right)$.

Lemma 4. If $\left\|u_{n}-u\right\|_{W_{5}} \rightarrow 0, x_{n} \rightarrow x,(n \rightarrow \infty)$, and $f(x, y)$ is continuous with respect to $x, y$ for $x \in[0,1], y \in$ $(-\infty,+\infty)$, then

$$
f\left(x_{n}, u_{n-1}\left(x_{n}\right)\right) \longrightarrow f(x, u(x)) \text { as } n \longrightarrow \infty .
$$

Proof. Since $\left\|u_{n}-u\right\|_{W_{5}} \rightarrow 0,(n \rightarrow \infty)$, by Lemma 3, we know that $u_{n}(x)$ is convergent uniformly to $u(x)$; therefore, the proof is complete.

\section{An Iterative Algorithm and Its Convergence}

In this section, the exact solution of (1) is given in the reproducing kernel space $W_{5}[0,1]$.

Theorem 5. If $\left\{x_{i}\right\}_{i=1}^{\infty}$ is dense on $[0,1]$ and $u(x) \in W_{5}[0,1]$ is the solution of (20), then $u(x)$ satisfies the form

$$
u(x)=\sum_{i=1}^{\infty} \sum_{k=1}^{i} \beta_{i k} F\left(x_{k}, u\left(x_{k}\right)\right) \overline{\psi_{i}}(x) .
$$

Proof. $u(x)$ can be expanded to the Fourier series in terms of normal orthogonal basis $\bar{\psi}_{i}(x)$ in $W_{2}^{5}[0,1]$ :

$$
\begin{aligned}
u(x) & =\sum_{i=1}^{\infty}\left\langle u(x), \bar{\psi}_{i}(x)\right\rangle_{W_{5}} \bar{\psi}_{i}(x) \\
& =\sum_{i=1}^{\infty} \sum_{k=1}^{i} \beta_{i k}\left\langle u(x), \psi_{k}(x)\right\rangle_{W_{5}} \bar{\psi}_{i}(x) \\
& =\sum_{i=1}^{\infty} \sum_{k=1}^{i} \beta_{i k}\left\langle u(x), L^{*} \varphi_{k}(x)\right\rangle_{W_{5}} \bar{\psi}_{i}(x) \\
& =\sum_{i=1}^{\infty} \sum_{k=1}^{i} \beta_{i k}\left\langle L u(x), \varphi_{k}(x)\right\rangle_{W_{1}} \bar{\psi}_{i}(x) \\
& =\sum_{i=1}^{\infty} \sum_{k=1}^{i} \beta_{i k}\left\langle F(x, u(x)), \varphi_{k}(x)\right\rangle_{W_{1}} \bar{\psi}_{i}(x) \\
& =\sum_{i=1}^{\infty} \sum_{k=1}^{i} \beta_{i k} f\left(x_{k}, u\left(x_{k}\right)\right) \bar{\psi}_{i}(x) .
\end{aligned}
$$


Remark 6. (i) If (20) is linear, that is, $F(x, u(x))=F(x)$, then the analytical solution of (20) can be obtained directly by (26).

(ii) If (20) is nonlinear, that is, $F$ depends on $u$, then the solution of (20) can be obtained by the following iterative method.

We construct an iterative sequence $u_{n}(x)$, putting any fixed $u_{0}(x) \in W_{5}[0,1]$,

$$
u_{n}(x)=\sum_{i=1}^{n} A_{i} \bar{\psi}_{i}(x),
$$

where

$$
\begin{aligned}
& A_{1}=\beta_{11} F\left(x_{1}, u_{0}\left(x_{1}\right)\right), \\
& A_{2}=\sum_{k=1}^{2} \beta_{2 k} F\left(x_{k}, u_{k-1}\left(x_{k}\right)\right), \\
& \vdots \\
& A_{n}=\sum_{k=1}^{n} \beta_{n k} F\left(x_{k}, u_{k-1}\left(x_{k}\right)\right) .
\end{aligned}
$$

Next, we prove that $u_{n}(x)$ in iterative formula (28) is convergent to the exact solution of (20).

Theorem 7. Suppose the following conditions are satisfied:

(i) $\left\|u_{n}\right\|_{W_{5}}$ is bounded;

(ii) $\left\{x_{i}\right\}_{i=1}^{\infty}$ is dense in $[0,1]$;

(iii) $f(x, y(x)) \in W_{1}[0,1]$ for any $y(x) \in W_{5}[0,1]$.

Then, $u_{n}(x)$ in iterative formula (28) converges to the exact solution $u(x)$ of $(20)$ in $W_{5}[0,1]$ and

$$
u(x)=\sum_{i=1}^{\infty} A_{i} \bar{\psi}_{i},
$$

where $A_{i}$ are given by (29).

Proof. (i) First, we will prove the convergence of $u_{n}(x)$.

By (28), we have

$$
u_{n+1}(x)=u_{n}(x)+A_{n+1} \bar{\psi}_{n+1}(x) .
$$

From the orthogonality of $\{\bar{\psi}(x)\}_{i=1}^{\infty}$, it follows that

$$
\begin{aligned}
\left\|u_{n+1}\right\|_{W_{5}}^{2} & =\left\|u_{n}\right\|_{W_{5}}^{2}+\left(A_{n+1}\right)^{2} \\
& =\left\|u_{n-1}\right\|_{W_{5}}^{2}+\left(A_{n}\right)^{2}+\left(A_{n+1}\right)^{2} \\
& =\cdots \\
& =\sum_{i=1}^{n+1}\left(A_{i}\right)^{2} .
\end{aligned}
$$

From boundedness of $\left\|u_{n}\right\|_{W_{5}}$, we have $\sum_{i=1}^{\infty}\left(A_{i}\right)^{2}<\infty$; that is, $\left\{A_{i}\right\} \in l^{2}(i=1,2, \ldots)$.
Let $m>n$; in view of $\left(u_{m}-u_{m-1}\right) \perp\left(u_{m-1}-u_{m-2}\right) \perp \cdots \perp$ $\left(u_{n+1}-u_{n}\right)$, it follows that

$$
\begin{aligned}
\| u_{m}- & u_{n} \|_{W_{5}}^{2} \\
& =\left\|u_{m}-u_{m-1}+u_{m-1}-u_{m-2}+\cdots+u_{n+1}-u_{n}\right\|_{W_{5}}^{2} \\
& \leq\left\|u_{m}-u_{m-1}\right\|_{W_{5}}^{2}+\cdots+\left\|u_{n+1}-u_{n}\right\|_{W_{5}}^{2} \\
& =\sum_{i=n+1}^{m}\left(A_{i}\right)^{2} \longrightarrow 0, \quad(m, n \longrightarrow \infty) .
\end{aligned}
$$

Considering the completeness of $W_{5}[0,1]$, there exists $u(x) \in$ $W_{5}[0,1]$, such that

$$
u_{n}(x) \stackrel{\|\cdot\|_{W_{5}}}{\longrightarrow} u(x), \quad \text { as } n \longrightarrow \infty .
$$

(ii) Second, we will prove that $u(x)$ is the solution of (20).

By Lemma 3 and (i) of Theorem 7, we know that $u_{n}(x)$ converge uniformly to $u(x)$. It follows that, on taking limits in (28), we have

$$
u(x)=\sum_{i=1}^{\infty} A_{i} \bar{\psi}_{i}
$$

Since

$$
\begin{aligned}
(L u)\left(x_{j}\right) & =\sum_{i=1}^{\infty} A_{i}\left\langle L \bar{\psi}_{i}(x), \varphi_{j}(x)\right\rangle_{W_{1}} \\
& =\sum_{i=1}^{\infty} A_{i}\left\langle\bar{\psi}_{i}(x), L^{*} \varphi_{j}(x)\right\rangle_{W_{5}} \\
& =\sum_{i=1}^{\infty} A_{i}\left\langle\bar{\psi}_{i}(x), \psi_{j}(x)\right\rangle_{W_{5}},
\end{aligned}
$$

it follows that

$$
\begin{aligned}
\sum_{j=1}^{n} \beta_{n j}(L u)\left(x_{j}\right) & =\sum_{i=1}^{\infty} A_{i}\left\langle\bar{\psi}_{i}(x), \sum_{j=1}^{n} \beta_{n j} \psi_{j}(x)\right\rangle_{W_{5}} \\
& =\sum_{i=1}^{\infty} A_{i}\left\langle\bar{\psi}_{i}(x), \bar{\psi}_{n}(x)\right\rangle_{W_{5}}=A_{n} .
\end{aligned}
$$

If $n=1$, then

$$
(L u)\left(x_{1}\right)=F\left(x_{1}, u_{0}\left(x_{1}\right)\right) .
$$

If $n=2$, then

$$
\begin{aligned}
& \beta_{21}(L u)\left(x_{1}\right)+\beta_{22}(L u)\left(x_{2}\right) \\
& =\beta_{21} F\left(x_{1}, u_{0}\left(x_{1}\right)\right)+\beta_{22} F\left(x_{2}, u_{1}\left(x_{2}\right)\right) .
\end{aligned}
$$

From (38) and (39), it is clear that $(L u)\left(x_{2}\right)=$ $F\left(x_{2}, u_{1}\left(x_{2}\right)\right)$; furthermore, it is easy to see by induction that

$$
(L u)\left(x_{j}\right)=F\left(x_{j}, u_{j-1}\left(x_{j}\right)\right) .
$$



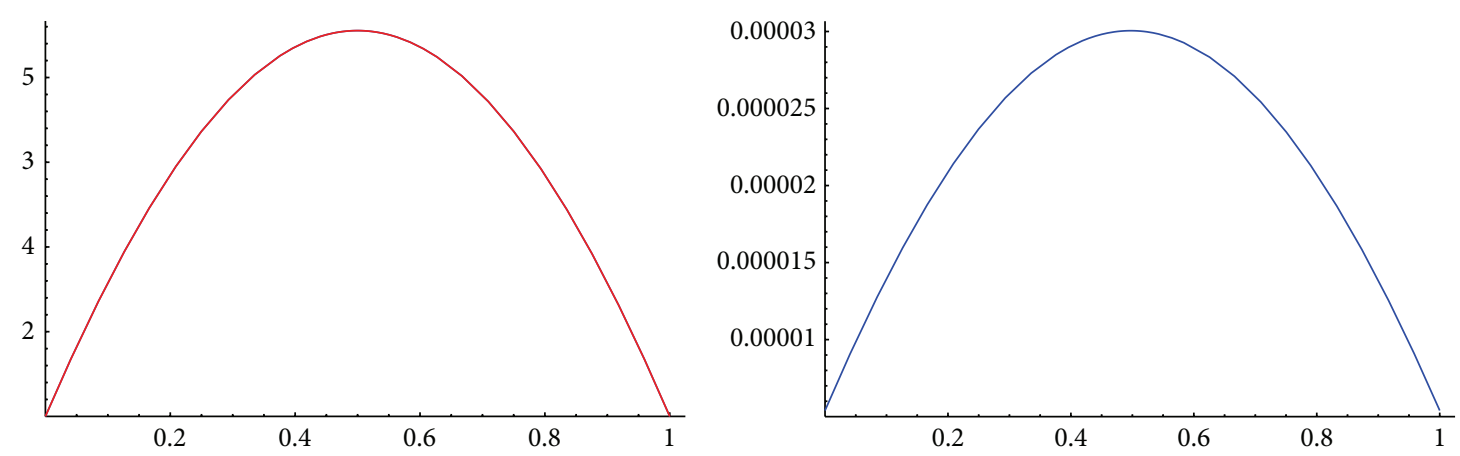

FIGURE 1: The congruent figure of $u(x)$ with $u_{100}(x)$ and $\left|u(x)-u_{100}(x)\right|$ for Example 1.

TABLE 1: The numerical results for Example 1.

\begin{tabular}{lcccc}
\hline Node & True solution $u(x)$ & Approximate solution $u_{100}(x)$ & Absolute error & Relative error \\
\hline 0.1 & 2.57757 & 2.57756 & $1.39670 E-5$ & $5.41865 E-6$ \\
0.2 & 3.85257 & 3.85255 & $2.08943 E-5$ & $5.42347 E-6$ \\
0.3 & 4.78900 & 4.78897 & $2.59723 E-5$ & $5.42333 E-6$ \\
0.4 & 5.36114 & 5.36111 & $2.90522 E-5$ & $5.41903 E-6$ \\
0.5 & 5.55357 & 5.55354 & $3.00501 E-5$ & $5.41096 E-6$ \\
0.6 & 5.36114 & 5.36111 & $2.89504 E-5$ & $5.40005 E-6$ \\
0.7 & 4.78900 & 4.78897 & $2.58036 E-5$ & $5.38810 E-6$ \\
0.8 & 3.85257 & 3.85255 & $2.07194 E-5$ & $5.37806 E-6$ \\
0.9 & 2.57757 & 2.57756 & $1.38544 E-5$ & $5.37499 E-6$ \\
1.0 & 1 & 0.999995 & $5.39775 E-6$ & $5.39775 E-6$ \\
\hline
\end{tabular}

Since $\left\{x_{i}\right\}_{i=1}^{\infty}$ is dense on interval $[0,1]$, for any $y \in[0,1]$, there exists subsequence $\left\{x_{n_{j}}\right\}$, such that $x_{n_{j}} \rightarrow y$, as $j \rightarrow$ $\infty$. Hence, let $j \rightarrow \infty$ in (40); by the convergence of $u_{n}(x)$ and Lemma 4, we have

$$
(L u)(y)=F(y, u(y))
$$

that is, $u(x)$ is the solution of $(20)$ and

$$
u(x)=\sum_{i=1}^{\infty} A_{i} \bar{\psi}_{i}
$$

where $A_{i}$ are given by (29).

From Lemma 3, we have Corollary 8.

Corollary 8. Assume that the conditions of Theorem 7 hold; then $u_{n}(x)$ in (28) satisfies $\left\|u_{n}-u\right\|_{C^{4}[0,1]} \rightarrow 0, n \rightarrow \infty$, where $u(x)$ is the solution of $(20)$.

\section{Numerical Experiment}

In this section, the method in the paper will be applied to some numerical examples. All computations are performed in Mathematica 5.0.
Example 1. Consider

$$
\begin{gathered}
u^{(4)}(x)=u^{2}(x)+f(x), \quad 0<x<1, \\
u(0)=u(1)=\frac{1}{2} \int_{0}^{1} x u(x) d x \\
u^{\prime \prime}(0)=u^{\prime \prime}(1)=\frac{3}{4} \int_{0}^{1} u^{\prime \prime}(x) d x
\end{gathered}
$$

The exact solution is $u(x)=1+(120 x / 7)-\left(90 x^{2} / 7\right)-$ $\left(60 x^{3} / 7\right)+\left(30 x^{4} / 7\right), f(x)=(720 / 7)-(1+(120 x / 7)-$ $\left.\left(90 x^{2} / 7\right)-\left(60 x^{3} / 7\right)+\left(30 x^{4} / 7\right)\right)^{2}$. The numerical results and the root-mean-square errors for the partial derivatives are displayed in Tables 1 and 2, respectively. The superimposed images of $u(x)$ with $u_{100}(x)$ and $\left|u(x)-u_{100}(x)\right|$ are displayed in Figure 1.

Example 2. Consider

$$
\begin{gathered}
u^{(4)}(x)=\sin [u(x)]+f(x), \quad 0<x<1, \\
u(0)=u(1)=\frac{2}{3} \int_{0}^{1} x u(x) d x, \\
u^{\prime \prime}(0)=u^{\prime \prime}(1)=\frac{5}{6} \int_{0}^{1}(x+3) u^{\prime \prime}(x) d x .
\end{gathered}
$$



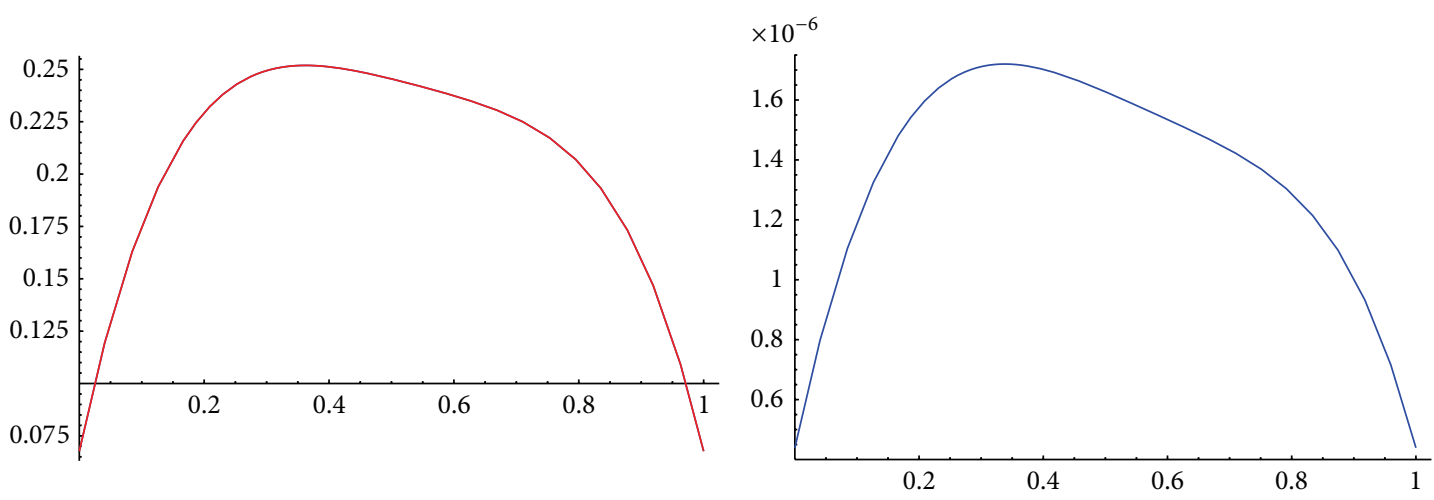

FIGURE 2: The congruent figure of $u(x)$ with $u_{100}(x)$ and $\left|u(x)-u_{100}(x)\right|$ for Example 2.

TABLE 2: The root-mean-square errors for the partial derivatives for Example 1.

\begin{tabular}{lccc}
\hline 1-order derivative & 2-order derivative & 3-order derivative & 4-order derivative \\
\hline $5.65090 E-5$ & $1.85920 E-4$ & $1.56359 E-4$ & $5.80200 E-4$ \\
\hline
\end{tabular}

TABLE 3: The numerical results for Example 2.

\begin{tabular}{lcccc}
\hline Node & True solution $u(x)$ & Approximate solution $u_{100}(x)$ & Absolute error & Relative error \\
\hline 0.1 & 0.175365 & 0.175366 & $1.19292 E-6$ & $6.80252 E-6$ \\
0.2 & 0.229289 & 0.229291 & $1.57610 E-6$ & $6.87384 E-6$ \\
0.3 & 0.249391 & 0.249393 & $1.71129 E-6$ & $6.86188 E-6$ \\
0.4 & 0.251217 & 0.251218 & $1.70213 E-6$ & $6.77554 E-6$ \\
0.5 & 0.245453 & 0.245455 & $1.62740 E-6$ & $6.63021 E-6$ \\
0.6 & 0.237419 & 0.237420 & $1.53473 E-6$ & $6.46421 E-6$ \\
0.7 & 0.226498 & 0.226500 & $1.43357 E-6$ & $6.32929 E-6$ \\
0.8 & 0.205517 & 0.205519 & $1.28776 E-6$ & $6.26595 E-6$ \\
0.9 & 0.160054 & 0.160055 & $1.00724 E-6$ & $6.29316 E-6$ \\
1 & 0.0676755 & 0.0676759 & $4.39185 E-7$ & $6.48958 E-6$ \\
\hline
\end{tabular}

TABLE 4: The root-mean-square errors for the partial derivatives for Example 2.

\begin{tabular}{lccc}
\hline 1-order derivative & 2-order derivative & 3-order derivative & 4 -order derivative \\
\hline $3.45285 E-6$ & $2.39862 E-5$ & $1.28849 E-4$ & $4.55038 E-4$ \\
\hline
\end{tabular}

The exact solution is $u(x)=(1 /(1769+2401 e))(36(1769-$ $8280 e^{x}-60 x(74+x(41+23 x))+e(2401+60 x(80+x(35+$ $23 x))))), f(x)=-\left(298080 e^{x} /(1769+2401 e)\right)-\sin [(1 /(1769+$ $2401 e))\left(36\left(1769-8280 e^{x}-60 x(74+x(41+23 x))+e(2401+\right.\right.$ $60 x(80+x(35+23 x)))))]$. Similar to Example 1, the numerical results are displayed in Tables 3 and 4 and Figure 2.

\section{Conclusions}

In summary, we use an iterative method to find the approximate solution of the nonlinear fourth-order equation with integral boundary conditions in the reproducing kernel space. Using this method, we obtain the sequence which is proved to converge to the exact solution uniformly.
Numerical results show that the method employed in the paper is valid. It is worthy to note that the new method can be used as a very accurate algorithm for solving linear and nonlinear integral boundary problems.

\section{Conflict of Interests}

The authors declare that there is no conflict of interests regarding the publication of this paper.

\section{Acknowledgment}

The research is supported by the Scientific Research Project of Heilongjiang Education Office (no. 12511155). 


\section{References}

[1] J. M. Gallardo, "Second-order differential operators with integral boundary conditions and generation of analytic semigroups," The Rocky Mountain Journal of Mathematics, vol. 30, no. 4, pp. 1265-1292, 2000.

[2] G. L. Karakostas and P. C. Tsamatos, "Multiple positive solutions of some Fredholm integral equations arisen from nonlocal boundary-value problems," Electronic Journal of Differential Equations, vol. 2002, pp. 1-17, 2002.

[3] A. Lomtatidze and L. Malaguti, "On a nonlocal boundary value problem for second order nonlinear singular differential equations," Georgian Mathematical Journal, vol. 7, no. 1, pp. 133154,2000

[4] X. M. Zhang, M. Q. Feng, and W. G. Ge, "Existence results for nonlinear boundary-value problems with integral boundary conditions in Banach spaces," Nonlinear Analysis: Theory, Methods and Applications, vol. 69, no. 10, pp. 3310-3321, 2008.

[5] P. Kang, Z. L. Wei, and J. J. Xu, "Positive solutions to fourthorder singular boundary value problems with integral boundary conditions in abstract spaces," Applied Mathematics and Computation, vol. 206, no. 1, pp. 245-256, 2008.

[6] M. G. Cui and Z. Chen, "The exact solution of nonlinear agestructured population model," Nonlinear Analysis: Real World Applications, vol. 8, no. 4, pp. 1096-1112, 2007.

[7] E. Capobianco, "Kernel methods and flexible inference for complex stochastic dynamics," Physica A: Statistical Mechanics and Its Applications, vol. 387, no. 16-17, pp. 4077-4098, 2008.

[8] Y. F. Zhou, Y. Lin, and M. Cui, "An efficient computational method for second order boundary value problems of nonlinear differential equations," Applied Mathematics and Computation, vol. 194, no. 2, pp. 354-365, 2007.

[9] F. Z. Geng and M. G. Cui, "Solving singular nonlinear secondorder periodic boundary value problems in the reproducing kernel space," Applied Mathematics and Computation, vol. 192, no. 2, pp. 389-398, 2007.

[10] M. G. Cui and F. Z. Geng, "Solving singular two-point boundary value problem in reproducing kernel space," Journal of Computational and Applied Mathematics, vol. 205, no. 1, pp. 6-15, 2007.

[11] F. Z. Geng and M. G. Cui, "Solving a nonlinear system of second order boundary value problems," Journal of Mathematical Analysis and Applications, vol. 327, no. 2, pp. 1167-1181, 2007.

[12] X. M. Zhang, M. Q. Feng, and W. G. Ge, "Symmetric positive solutions for $p$-Laplacian fourth-order differential equations with integral boundary conditions," Journal of Computational and Applied Mathematics, vol. 222, no. 2, pp. 561-573, 2008.

[13] Y. L. Wang, X. J. Cao, and X. N. Li, "A new method for solving singular fourth-order boundary value problems with mixed boundary conditions," Applied Mathematics and Computation, vol. 217, no. 18, pp. 7385-7390, 2011.

[14] X. M. Zhang and W. G. Ge, "Symmetric positive solutions of boundary value problems with integral boundary conditions," Applied Mathematics and Computation, vol. 219, no. 8, pp. 35533564, 2012.

[15] M. G. Cui and Y. Z. Lin, Nonlinear Numercial Analysis in the Reproducing Kernel Space, Nova Science, New York, NY, USA, 2008. 


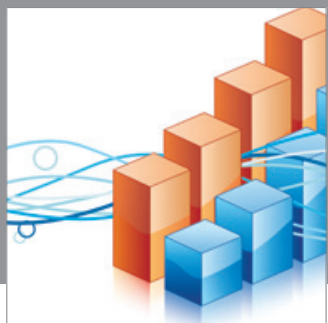

Advances in

Operations Research

mansans

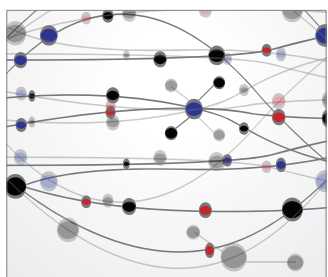

The Scientific World Journal
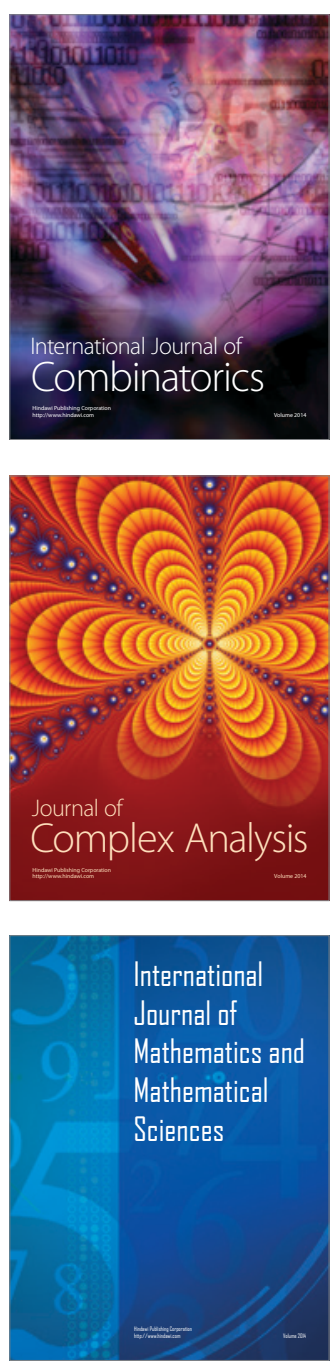
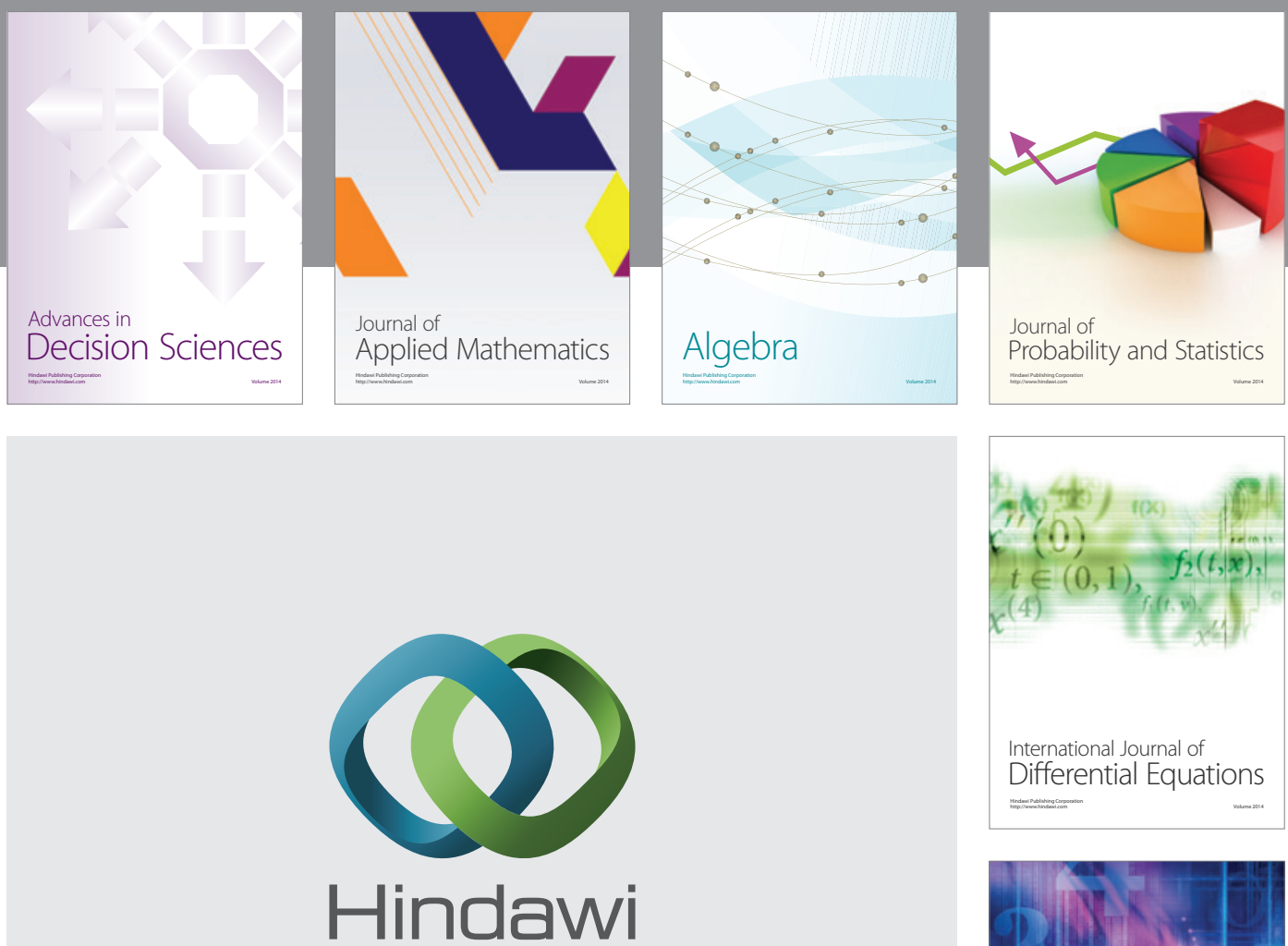

Submit your manuscripts at http://www.hindawi.com
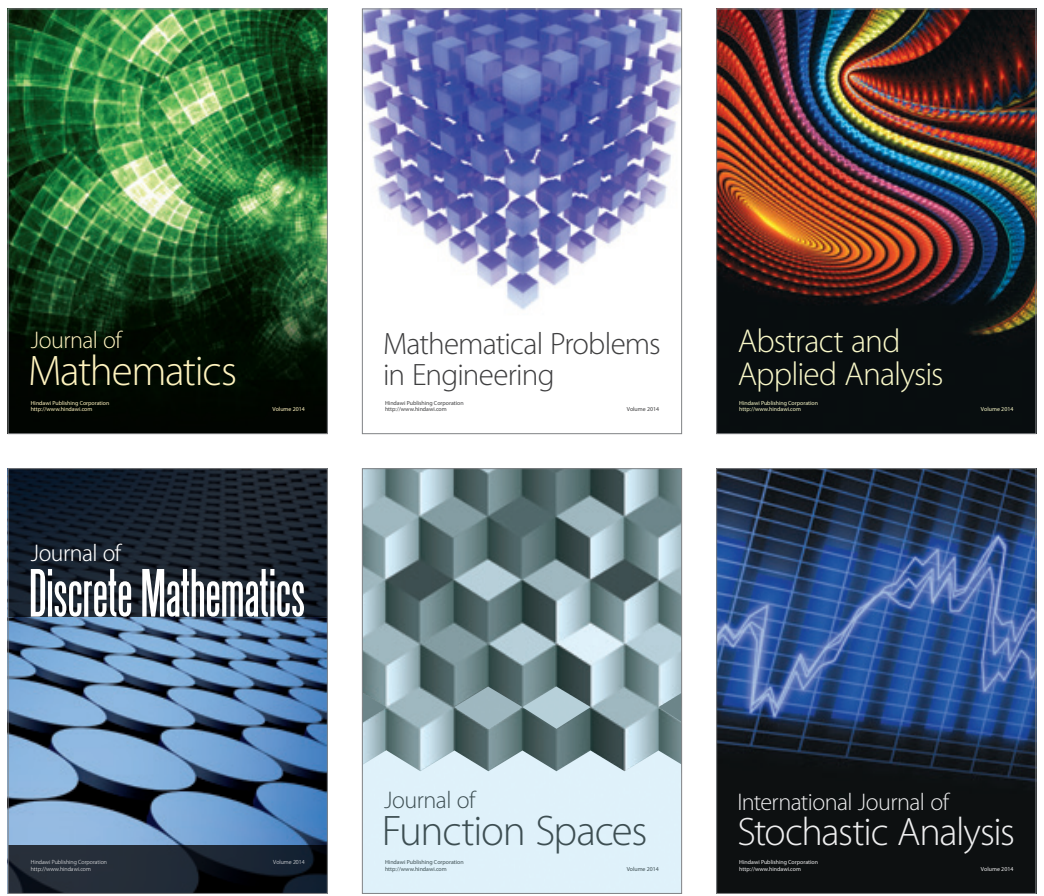

Journal of

Function Spaces

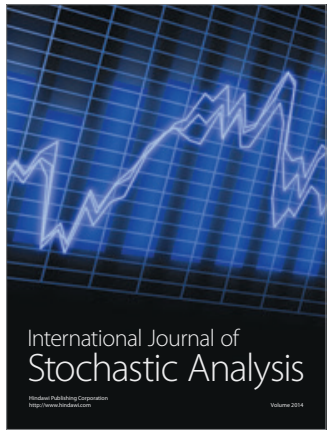

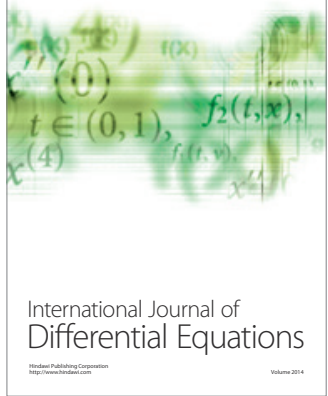
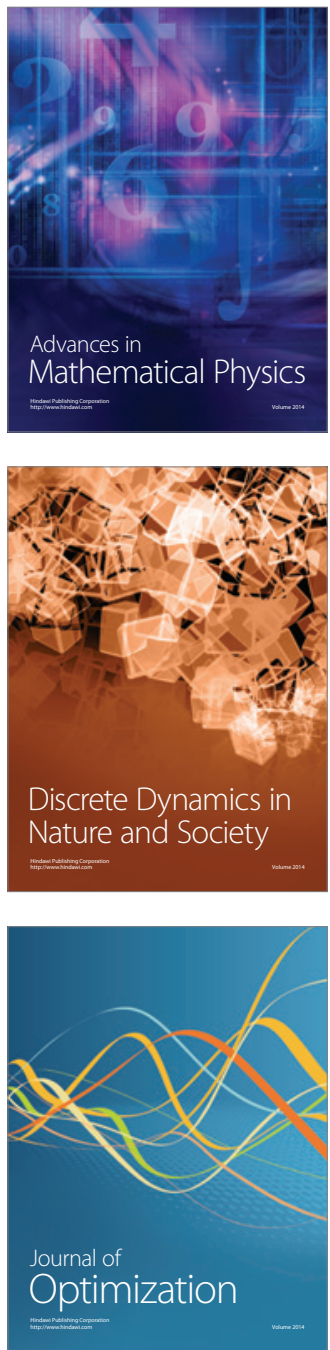